\title{
Porphyromonas bennonis sp. nov., isolated from human clinical specimens
}

\begin{abstract}
Correspondence
Paula H. Summanen carlsonph@aol.com
\end{abstract}

\author{
Paula H. Summanen, ${ }^{1}$ Paul A. Lawson ${ }^{2}$ and Sydney M. Finegold ${ }^{3,4,5}$ \\ ${ }^{1}$ Research Service, Anaerobe Laboratory, Building 304, Room E3-237, Veterans Affairs (VA) \\ West Los Angeles Healthcare Center, 11301 Wilshire Boulevard, Los Angeles, CA 90073, USA \\ ${ }^{2}$ Department of Botany and Microbiology, University of Oklahoma, Norman, OK, USA \\ ${ }^{3}$ Infectious Diseases Section, VA West Los Angeles Healthcare Center, Los Angeles, CA, USA \\ ${ }^{4}$ Department of Medicine, University of California at Los Angeles School of Medicine, Los Angeles, \\ CA, USA \\ ${ }^{5}$ Department of Microbiology, Immunology and Molecular Genetics, University of California at \\ Los Angeles School of Medicine, Los Angeles, CA, USA
}

\begin{abstract}
During our investigation of the bacteriology of human wound infections and abscesses, a novel anaerobic, non-spore-forming, Gram-negative bacillus was frequently isolated. On the basis of morphological and biochemical criteria, the strains were tentatively identified as belonging to the family Bacteroidaceae, but they did not appear to correspond to any recognized species of this family. Comparative 16S rRNA gene sequencing showed that the 14 novel strains were genotypically homogeneous and confirmed their placement in the genus Porphyromonas. Sequence divergence values $>10 \%$ with respect to reference Porphyromonas species demonstrated that the strains isolated represent a novel species. On the basis of biochemical criteria and phylogenetic considerations, it is proposed that these strains isolated from human sources should be assigned to a novel species of the genus Porphyromonas, named Porphyromonas bennonis sp. nov., with WAL $1926 C^{\top}$ (=ATCC BAA-1629 ${ }^{\top}=$ CCUG $55979^{\top}$ ) as the type strain.
\end{abstract}

Members of the genus Porphyromonas are indigenous bacterial flora in the oral cavity of humans and animals; many species are also found in the urogenital and intestinal tracts. Some species are considered true pathogens and are associated with human or animal infections. Porphyromonas species have been isolated from oral infections and from many infections throughout the body. Porphyromonas species of animal origin have been encountered in humans with animal-bite infections. During our investigation of the bacteriology of human wound infections and abscesses (Finegold et al., 2008), a novel anaerobic, non-spore-forming, Gram-negative bacillus related distantly to Porphyromonas somerae (Summanen et al., 2005) was frequently isolated. Comparative $16 \mathrm{~S}$ rRNA gene sequencing studies demonstrated that the 14 novel strains were genotypically homogeneous, clustering within the phylogeny of the genus Porphyromonas. In this polyphasic taxonomic study, we describe the genotypic and phenotypic characterization of this organism, which represents a novel species of the genus Porphyromonas.

The GenBank/EMBL/DDBJ accession number for the $16 \mathrm{~S}$ rRNA gene sequence of strain WAL $1926 C^{\top}$ is EU414673.
Fourteen strains isolated at the VA Wadsworth Medical Center were included in this study (Table 1). All strains were characterized by using $16 \mathrm{~S}$ rRNA gene sequencing and conventional biochemical methods, unless otherwise indicated. $P$. somerae ATCC BAA- $1230^{\mathrm{T}}$ was included in the biochemical and molecular characterizations. The strains were maintained at $-70{ }^{\circ} \mathrm{C}$ in double-strength skimmed milk before characterization. The strains were grown at $37{ }^{\circ} \mathrm{C}$ on Brucella blood agar (Anaerobe Systems) or CDC blood agar (Becton Dickinson) under anaerobic conditions $\left(\mathrm{N}_{2}: \mathrm{CO}_{2}: \mathrm{H}_{2}, 90: 5: 5\right)$. All tests were performed on 24$72 \mathrm{~h}$ pure cultures, unless otherwise indicated.

The strains were characterized by using conventional biochemical tests (Durmaz et al., 1995; Jousimies-Somer, 1995; Jousimies-Somer et al., 2002; Holdeman et al., 1977), including the assessment of susceptibility to special potency identification discs, the fermentation of pre-reduced, anerobically sterilized biochemicals (Anaerobe Systems), GLC of metabolic end products, API ZYM and Rapid ID 32A (bioMérieux) and Rosco Diagnostic Tablets (Rosco). Pigment production was tested on laked blood agar (Anaerobe Systems). The production of $\beta$-lactamase was assessed by using nitrocefin-impregnated discs (Cefinase; 
Table 1. Novel strains included in this study

\begin{tabular}{|llc|}
\hline Strain & \multicolumn{1}{c|}{ Isolation source (history) } & Cell count (c.f.u. $\mathbf{~ m l}^{\mathbf{- 1}}$ ) \\
\hline WAL 1706J & Infected pilonidal cyst (negative) & $10^{5}$ \\
WAL 1773I & Spontaneous groin abscess (negative) & $10^{8}$ \\
WAL 1804E & Axillary abscess (2 year history of lump) & $10^{7}$ \\
WAL 1860J & Buttock abscess (multiple episodes of rectal fistula) & $10^{7}$ \\
WAL 1871H & Breast abscess (diabetes mellitus) & $10^{5}$ \\
WAL 1875-12 & Perirectal abscess (negative) & $10^{8}$ \\
WAL 1894-1 & Thumb paronychia (diabetes mellitus) & $10^{7}$ \\
WAL 1896H & Spontaneous breast abscess (negative) & $10^{7}$ \\
WAL 1926C & Spontaneous shoulder abscess (diabetes mellitus) & $10^{7}$ \\
WAL 1980J & Spontaneous buttock abscess (diabetes mellitus) & $10^{8}$ \\
WAL 1998F & Vaginal abscess (negative) & $10^{8}$ \\
WAL 2044F & Spontaneous breast abscess (diabetes mellitus) & $10^{5}$ \\
WAL 2045I & Spontaneous lower back abscess (negative) & $10^{3}$ \\
WAL 2083H & Groin abscess (intravenous drug abuser) & $10^{6}$ \\
& & \\
\hline
\end{tabular}

Becton Dickinson). In all tests with pre-reduced, anerobically sterilized biochemicals, supplementation with haemin $(0.001 \%)$ and rabbit serum $(1 \%)$ was used to obtain growth. For cellular fatty acid analyses, the cells were grown on Brucella blood agar for $48 \mathrm{~h}$. Growth was collected in $0.7 \%(\mathrm{w} / \mathrm{v}) \quad \mathrm{MgSO}_{4}$ and harvested by centrifugation $(5000 \mathrm{~g}$ for $20 \mathrm{~min}$ ); the pellets were processed immediately or kept frozen at $-20{ }^{\circ} \mathrm{C}$. Methyl ester extraction was carried out according to the manufacturer's procedure (MIDI; Microbial ID) and the analysis was performed by using a Microbial Identification System (model 5898A; Hewlett Packard) gas chromatograph, with software from MIDI. The corresponding library (ANAEROBE, version Moore 5.0) was used in successive analyses. All biochemical tests were performed in duplicate. The $\mathrm{KOH}$ string test was performed as described by Halebian et al. (1981).

Genomic DNA was extracted and purified from bacterial cells in the mid-exponential growth phase, using a QIAamp DNA Mini kit (Qiagen). 16S rRNA gene fragments were amplified by means of a PCR with universal primers. The PCR products were purified by using a QIAquick gel extraction kit (Qiagen). The purified PCR products were sequenced directly with BigDye Terminator cycle sequencing kits (Biotech Diagnostics) on an ABI 3100 Avant Genetic System (Applied Biosystems). The sequencing data were analysed by comparing the consensus sequences with sequences obtained from GenBank, using the Ribosomal Database Project (RDP-II) (Maidak et al., 2001) and BLAST software (Benson et al., 1999): similarity (\%) with respect to other sequences was determined. Closely related sequences were retrieved from GenBank and were aligned with the newly determined sequences by using the program CLUSTAL W (Thompson et al., 1994). The phylogenetic tree was constructed by using the CLUSTAL w method, with the SeqBuilder and MegAlign program modules of LASERGENE 7 (DNASTAR); bootstrap analysis was also performed with MegAlign. The $\mathrm{G}+\mathrm{C}$ content $(\mathrm{mol} \%)$ of the DNA was determined by using HPLC according to the method of Mesbah et al. (1989).

The unidentified isolates were recovered from clinical infections as a part of the infecting flora together with other anaerobes or aerobes, generally at high counts $\left(>10^{5}\right.$ c.f.u. $\left.\mathrm{ml}^{-1}\right)$ (Table 1). The mean number of accompanying aerobes for each c.f.u. of the unidentified isolates was 1.8 and that for anaerobes was 4.1. Colonies grown on anaerobic blood agar plates for 48-72 $\mathrm{h}$ were small (0.2-0.5 mm in diameter), circular, entire, low convex, opaque and slightly $\beta$-haemolytic. At an early stage of growth, the colonies did not show pigmentation or fluorescence under long-wave UV light (366 nm); the colour of colonies ranged from grey to cream. After more prolonged incubation ( $\geqslant 10$ days), a light beige to tan pigment developed; fluorescence remained negative. On Brucella blood agar, cells were generally Gram-negative, $0.7-1.0 \times 1.0-6.0 \mu \mathrm{m}$ in size and pleomorphic, occasionally with long filaments and swellings. Occasionally, cells appeared Gram-positive in young cultures; however, the string test was positive, indicating that they were Gramnegative. The novel isolates were all resistant to colistin and kanamycin special potency identification discs, and nine of the 14 strains tested were sensitive to the vancomycin disc. All of the isolates studied were lipase-, indole- and nitratenegative and sensitive to bile. Catalase results were variable, with six of the 14 strains registering as catalase-positive. All strains hydrolysed gelatin. All were asaccharolytic and produced acetic acid and succinic acid, and occasionally trace amounts of iso-valeric acid and lactic acid, as metabolic end products. All strains were positive for alkaline phosphatase, esterase (weakly positive), leucine arylamidase, chymotrypsin (weakly positive), acid phosphatase, naphthol-AS-BI-phosphohydrolase, $\beta$-galactosidase, $\alpha$-glucosidase and $N$-acetyl- $\beta$-glucosaminidase, but were negative for cystine arylaminidase, trypsin, $\alpha$-galactosidase, $\beta$-glucuronidase, $\beta$-glucosidase, $\alpha$-mannosidase and $\alpha$-fucosidase (in the API ZYM system). Esterase lipase, 
lipase and valine arylamidase activities were variable in the API ZYM test. Rosco diagnostic tablets indicated that the strains were positive for $\alpha$-glucosidase, $N$-acetyl- $\beta$-glucosaminidase and ONPG, but were negative for $\alpha$-fucosidase, trypsin, urease and aesculin. In the Rapid ID 32A system, $\beta$ galactosidase, $\beta$-galactosidase phosphate, $\alpha$-glucosidase, $N$ acetyl- $\beta$-glucosaminidase, alkaline phosphatase, leucyl glycine arylamidase and alanine arylamidase were positive, whereas urease, arginine dihydrolase, $\alpha$-galactosidase, $\beta$ glucosidase, $\alpha$-arabinosidase, $\beta$-glucuronidase, mannosidase and raffinose fermentation, $\alpha$-fucosidase and pyroglutamic acid arylamidase gave negative results. Other reactions with Rapid ID 32A were variably negative or weakly positive. $\beta$ Lactamase was produced by two of 13 strains (15\%). The main cellular fatty acids detected in the novel organism belonged to summed feature $5\left(\mathrm{C}_{15: 0} \mathrm{DMA}\right.$ and/or $\mathrm{C}_{14: 0} 3$ $\mathrm{OH}$, which could not be separated by the method used; 23 $40 \%$ of the total fatty acids). Other cellular fatty acids (detected at levels in the range $9-15 \%$ of total fatty acids) included $\mathrm{C}_{12: 0}, \mathrm{C}_{14: 0}$ and $\mathrm{C}_{16: 0}$. Cluster analysis of the principal components showed a Euclidian distance of $<10$ between the unidentified strains, thus indicating that they belong to the same species. Biochemical tests that are useful for differentiating this novel organism from other species and genera are listed in Table 2. The key characteristics that serve to differentiate this novel organism from other Porphyromonas species are the negative $\alpha$-fucosidase results, the positive $N$-acetyl- $\beta$-glucosaminidase and $\beta$-galactosidase results and the production of acetic and succinic acids as metabolic end products. In addition, the fact that the novel organism produces only weakly pigmented, small colonies on blood agar is also useful for differentiation (except with respect to Porphyromonas catoniae, which is non-pigmented; Willems \& Collins, 1995).

In this polyphasic taxonomic study, the unidentified strains were related closely to each other genetically, as demonstrated by the 16S rRNA gene sequence similarities (99$100 \%)$. Phylogenetic analysis demonstrated that the novel organism represents a distinct line of descent within the genus Porphyromonas, as exemplified by strain WAL $1926^{\mathrm{T}}$ (Fig. 1). The mean interspecies similarity within the Porphyromonas cluster is approximately $87 \%$ (Paster et al., 1994); in GenBank, the closest match to the novel organism was P. somerae WAL $6690^{\mathrm{T}}$, at $89-90 \%$ sequence similarity. There is no precise correlation between $16 \mathrm{~S}$ rRNA gene sequence divergence (\%) and species delineation, but it is generally recognized that divergence values of $3 \%$ or more

Table 2. Characteristics of $P$. bennonis sp. nov. that serve to differentiate it from recognized species of the genus Porphyromonas

Taxa: 1, P. asaccharolytica; 2, P. endodontalis; 3, P. gingivalis; 4, P. catoniae; 5, P. somerae; 6, P. uenonis; 7, P. bennonis sp. nov. ( $n=14)$. All taxa are negative for lipase activity. Data for taxa 1-4 are from Jousimies-Somer et al. (2002); data for taxon 5 are from Summanen et al. (2005) and those for taxon 6 are from Finegold et al. (2004). Symbols:,$+ \geqslant 90 \%$ of the strains are positive;,$- \geqslant 10 \%$ of the strains are negative; D, $11-89 \%$ of the strains are positive; $\mathrm{w}$, weakly positive reaction.

\begin{tabular}{|c|c|c|c|c|c|c|c|}
\hline Characteristic & 1 & 2 & 3 & 4 & 5 & 6 & 7 \\
\hline Fluorescence & + & + & - & - & $\mathrm{D}$ & + & - \\
\hline Catalase & - & - & - & - & - & - & $\mathrm{D}$ \\
\hline \multicolumn{8}{|l|}{ Enzyme activity (commercial tests) ${ }^{*}$} \\
\hline$\alpha$-Fucosidase & + & - & - & + & - & - & - \\
\hline$N$-Acetyl- $\beta$-glucosaminidase & - & - & + & + & + & - & + \\
\hline Chymotrypsin & - & - & - & $\mathrm{D}$ & + & - & + \\
\hline Trypsin & - & - & + & $\mathrm{D}$ & - & - & - \\
\hline \multicolumn{8}{|l|}{ Fermentation of: $\ddagger$} \\
\hline Glucose & - & - & - & + & $\mathrm{W}$ & - & - \\
\hline
\end{tabular}

${ }^{\star}$ Reaction in the API ZYM system (bioMérieux) or with Rosco diagnostic tablets. Results obtained with these two systems are not always identical to each other (see footnote $\dagger$ ).

$\dagger$ Negative in the API ZYM system, but positive in the Rosco ONPG test.

$\$$ Fermentation of most other carbohydrates has been reported as negative.

$\$ A$, Acetic acid; P, propionic acid; IB, isobutyric acid; B, butyric acid; IV, isovaleric acid; V, valeric acid; L, lactic acid; S, succinic acid; PA, phenylacetic acid. Upper-case letters indicate major metabolic products from peptone-yeast extract-glucose (PYG) and lower-case letters indicate minor products. 


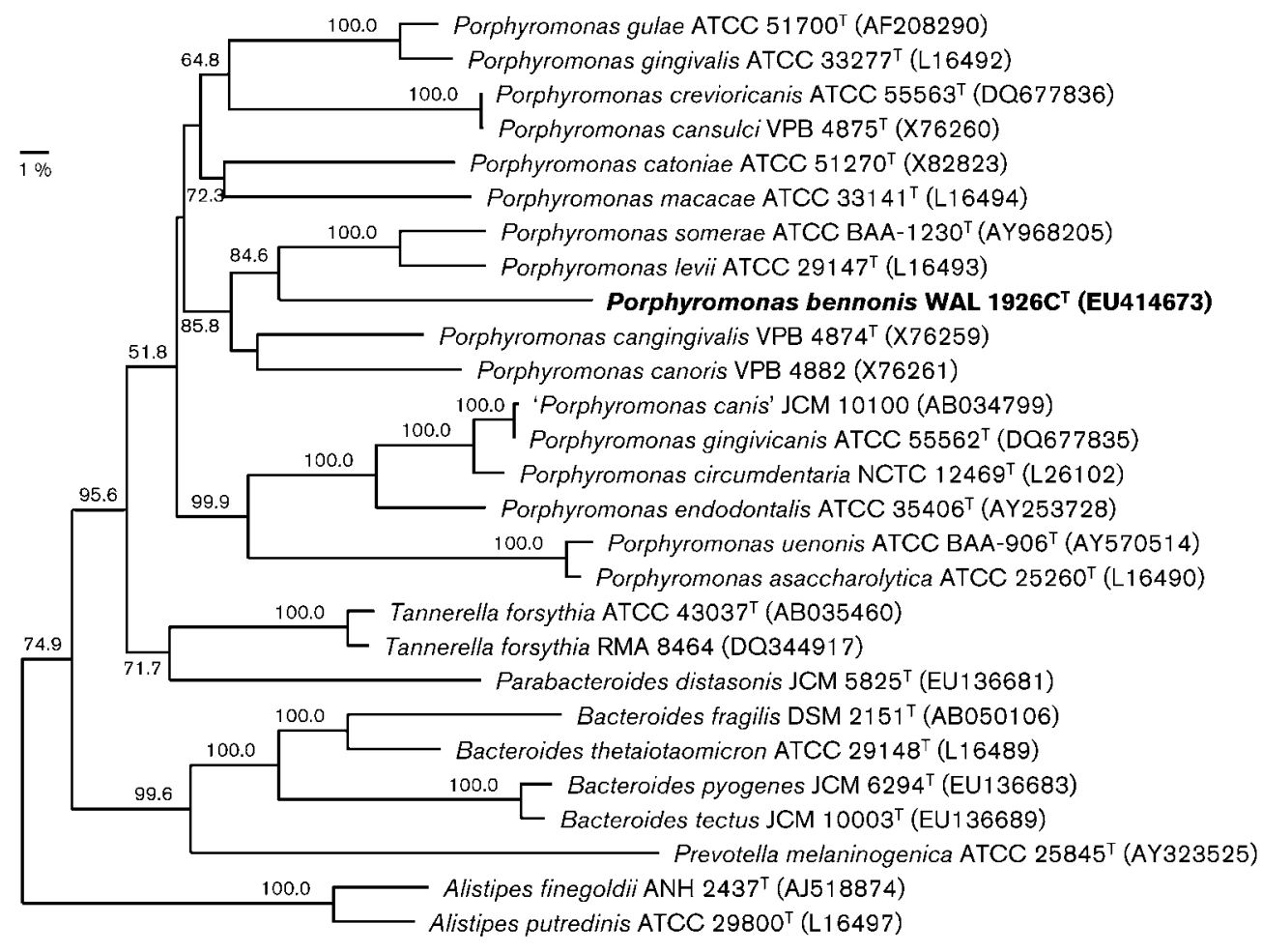

Fig. 1. Unrooted phylogenetic tree, based on $16 \mathrm{~S}$ rRNA gene sequences, showing the position of $P$. bennonis sp. nov. within the Bacteroides subgroup of the phylum Cytophaga-Flavobacterium-Bacteroides. Bootstrap percentages (based on 1000 replications) are given at branching points. Bar, $1 \%$ sequence divergence.

are significant (Stackebrandt \& Goebel, 1994). Support for the distinctiveness of the unknown bacterium was also very evident from the phenotypic analyses.

Whilst the data from the phylogenetic analysis were consistent with the view that the unknown organism belongs to the genus Porphyromonas, several of the organism's phenotypic characteristics did not conform to the description of the genus (Willems \& Collins, 1995). Rather like the members of the genus Porphyromonas, most of the novel isolates were sensitive to the special potency disc containing vancomycin. In addition, like most other human Porphyromonas species, the unknown organism was asaccharolytic. The organism produced very slightly pigmented colonies on laked blood agar only after prolonged incubation, whereas pigment production in most Porphyromonas species is evident after 2-4 days. Unlike most Porphyromonas species, which produce complex metabolic end-product profiles that include acetic, propionic, isobutyric, butyric and isovaleric acids, the novel organism produced moderate amounts of acetic and succinic acids, occasionally with trace amounts of isovaleric acid and lactic acid. The cellular fatty acid profile of the novel organism is unlike that of any other Porphyromonas species, in that predominating cellular fatty acids described for the latter are iso- $\mathrm{C}_{15: 0}$, iso- $\mathrm{C}_{17: 0} 3-\mathrm{OH}$ and anteiso- $\mathrm{C}_{15: 0}$ (Brondz et al., 1989; Shah \& Collins, 1988;
Brondz \& Olsen, 1991; Könönen et al., 1996; Summanen et al., 2005), whereas the main cellular fatty acid(s) in the former was $\mathrm{C}_{15: 0}$ DMA and/or $\mathrm{C}_{14: 0} 3-\mathrm{OH}(23-40 \%$ of total fatty acids). In the case of iso- $\mathrm{C}_{15: 0}$, iso- $\mathrm{C}_{17: 0} 3-\mathrm{OH}$ and anteiso- $\mathrm{C}_{15: 0}$, the levels detected were very low $(<1 \%)$ or non-existent. Furthermore, the DNA G $+\mathrm{C}$ content of the unknown organism $(58 \mathrm{~mol} \%)$ is somewhat higher than those of recognized Porphyromonas species (44-55 mol\%).

With an accurate identification scheme, this hithertounidentified organism may prove to be more common in clinical specimens than has been appreciated. During our comprehensive microbiological evaluation of infections of skin and soft tissue (Finegold et al., 2008), the novel organism was the most frequently isolated anaerobic, Gram-negative rod: 5\% (19 of 394) of specimens yielded this organism. Even though the novel organism was also isolated from areas above the diaphragm, we found that the isolates were predominantly recovered from patients with chronic infections of skin or soft tissue in the perirectal/ buttock/groin regions $(63 \%)$. The most common predisposing condition in the patients with skin and soft-tissue infections was diabetes mellitus. There were several relatively serious infections in which the unidentified organism was part of the infecting flora at high counts (Table 1). The full extent of the virulence of this newly identified organism remains to be determined, as it was 
always isolated from mixed culture. It is notable that $15 \%$ of the novel strains were $\beta$-lactamase producers; in the genus Porphyromonas, this is a trait found only in $P$. somerae (Summanen et al., 2005), Porphyromonas uenonis (Finegold et al., 2004) and perhaps also $P$. catoniae (Könönen et al., 1996). In mixed infections, $\beta$-lactamaseproducing species may protect other penicillin-susceptible bacteria by releasing free $\beta$-lactamase into their immediate environment (Brook, 2004).

Our studies indicate that this novel organism isolated from human sources clusters phylogenetically within the genus Porphyromonas, but is phenotypically and genetically different from recognized Porphyromonas species. We therefore propose the name Porphyromonas bennonis sp. nov. for this novel species.

\section{Description of Porphyromonas bennonis sp. nov.}

Porphyromonas bennonis (ben.no'nis. N.L. masc. gen. n. bennonis of Benno, in honour of the contemporary Japanese microbiologist Yoshimi Benno, who has contributed significantly to our knowledge of anaerobic bacteria).

Cells are Gram-negative, non-spore-forming rods, 0.7$1.0 \times 1.0-6.0 \mu \mathrm{m}$ in size. Obligately anaerobic. Optimum temperature for growth is $37^{\circ} \mathrm{C}$. Colonies incubated on blood agar for 2 days are small, circular, entire, convex and slightly $\beta$-haemolytic. Colonies are grey to cream; after 10 days incubation, colonies are weakly pigmented (beige to $\tan$ ) and show no red fluorescence under long-wave UV light. Gelatin-positive and bile-sensitive. Indole-, nitrateand lipase-negative. Urease- and aesculin-negative. Catalase-variable. Asaccharolytic. The major fermentation products from glucose metabolism are acetic acid and succinic acid. The major cellular fatty acids are $\mathrm{C}_{15: 0}$ DMA and/or $\mathrm{C}_{14: 0} 3-\mathrm{OH}, \mathrm{C}_{12: 0}, \mathrm{C}_{14: 0}$ and $\mathrm{C}_{16: 0}$. Positive for alkaline phosphatase, esterase (weakly positive), leucine arylamidase, chymotrypsin (weakly positive), acid phosphatase, naphthol-AS-BI-phosphohydrolase, $\beta$-galactosidase, $\alpha$-glucosidase and $N$-acetyl- $\beta$-glucosaminidase, but negative for cystine arylaminidase, trypsin, $\alpha$-galactosidase, $\beta$-glucuronidase, $\beta$-glucosidase, $\alpha$-mannosidase and $\alpha$ fucosidase (API ZYM system). Esterase lipase, lipase and valine arylamidase activities are variable with the API ZYM system. With Rosco diagnostic tablets, the strains are positive for $\alpha$-glucosidase, $N$-acetyl- $\beta$-glucosaminidase and ONPG, but negative for $\alpha$-fucosidase, trypsin, urease and aesculin. Positive for $\beta$-galactosidase, $\beta$-galactosidase phosphate, $\alpha$-glucosidase, $N$-acetyl- $\beta$-glucosaminidase, alkaline phosphatase, leucyl glycine arylamidase and alanine arylamidase, and negative for urease, arginine dihydrolase, $\alpha$-galactosidase, $\beta$-glucosidase, $\alpha$-arabinosidase, $\beta$-glucuronidase, mannosidase, raffinose fermentation, $\alpha$-fucosidase and pyroglutamic acid arylamidase (Rapid ID 32A). $\beta$-Lactamase is produced by $15 \%$ of the strains. The DNA $\mathrm{G}+\mathrm{C}$ content of the type strain is $58 \mathrm{~mol} \%$.
The type strain, WAL $1926 C^{\mathrm{T}}$ (=ATCC BAA-1629 $=$ CCUG $55979^{\mathrm{T}}$ ), and other strains were isolated in mixed culture from various skin and soft-tissue infections of nonoral origin, mainly from abscesses in the buttock and groin areas. Probably part of the indigenous bowel flora.

\section{Acknowledgements}

This work was supported by US Department of Defense grant W81XWH-0510134 and by VA Merit Review funds.

\section{References}

Benson, D. A., Boguski, M. S., Lipman, D. J., Ostell, J., Ouellette, B. F., Rapp, B. A. \& Wheeler, D. L. (1999). GenBank. Nucleic Acids Res 27, 12-17.

Brondz, I. \& Olsen, I. (1991). Multivariate analyses of cellular fatty acids in Bacteroides, Prevotella, Porphyromonas, Wolinella, and Campylobacter spp. J Clin Microbiol 29, 183-189.

Brondz, I., Carlsson, J., Sjöström, M. \& Sundqvist, G. (1989). Significance of cellular fatty acids and sugars in defining the genus Porphyromonas. Int J Syst Bacteriol 39, 314-318.

Brook, I. (2004). $\beta$-Lactamase-producing bacteria in mixed infections. Clin Microbiol Infect 10, 777-784.

Durmaz, B., Jousimies-Somer, H. R. \& Finegold, S. M. (1995). Enzymatic profiles of Prevotella, Porphyromonas, and Bacteroides species obtained with the API ZYM system and Rosco diagnostic tablets. Clin Infect Dis 20, S192-S194.

Finegold, S. M., Väisänen, M. L., Rautio, M., Eerola, E., Summanen, P., Molitoris, D., Song, Y., Liu, C. \& Jousimies-Somer, H. (2004). Porphyromonas uenonis sp. nov., a pathogen for humans distinct from $P$. asaccharolytica and P. endodontalis. J Clin Microbiol 42, 5298-5301.

Finegold, S. M., Talan, D., Hiyama, S., Rowlinson, M.-C., Summanen, P., Molitoris, D., Liu, C.-X., Ulger, N., Wooton, J. \& other authors (2008). Analysis of 400 surgical and traumatic wound infections and closed abscesses by molecular techniques for development of a real-time PCR procedure. In Abstracts of the 108th General Meeting of the American Society for Microbiology, Boston, MA, 1-5 June 2008. Washington, DC: American Society for Microbiology.

Halebian, S., Harris, B., Finegold, S. M. \& Rolfe, R. D. (1981). Rapid method that aids in distinguishing Gram-positive from Gramnegative anaerobic bacteria. J Clin Microbiol 13, 444-448.

Holdeman, L. V., Cato, E. P. \& Moore, W. E. C. (1977). Anaerobe Laboratory Manual. Blacksburg, VA: Virginia Polytechnic Institute and State University.

Jousimies-Somer, H. R. (1995). Update on the taxonomy and the clinical and laboratory characteristics of pigmented anaerobic Gramnegative rods. Clin Infect Dis 20, S187-S191.

Jousimies-Somer, H. R., Summanen, P., Citron, D., Baron, E., Wexler, H. M. \& Finegold, S. M. (2002). Wadsworth-KTL Anaerobic Bacteriology Manual, 6th edn. Belmont, CA: Star Publishing.

Könönen, E., Väisänen, M.-L., Finegold, S. M., Heine, R. \& JousimiesSomer, H. (1996). Cellular fatty acid analysis and enzyme profiles of Porphyromonas catoniae - a frequent colonizer of the oral cavity in children. Anaerobe 2, 329-335.

Maidak, B. L., Cole, R. J., Lilburn, T. G., Jr, Parker, C. T., Saxman, R., Farris, R. J., Garrity, G. M., Olsen, G. J., Schmidt, T. M. \& Tiedje, J. M. (2001). The RDP-II (Ribosomal Database Project). Nucleic Acids Res 29, 173-174. 
Mesbah, M., Premachandran, U. \& Whitman, W. B. (1989). Precise measurement of the $\mathrm{G}+\mathrm{C}$ content of deoxyribonucleic acid by highperformance liquid chromatography. Int J Syst Bacteriol 39, 159-167.

Paster, B. J., Dewhirst, F. E., Olsen, I. \& Fraser, G. J. (1994). Phylogeny of Bacteroides, Prevotella, and Porphyromonas spp. and related bacteria. J Bacteriol 176, 725-732.

Shah, H. N. \& Collins, M. D. (1988). Proposal for reclassification of Bacteroides asaccharolyticus, Bacteroides gingivalis, and Bacteroides endodontalis in a new genus, Porphyromonas. Int J Syst Bacteriol 38, 128-131.

Stackebrandt, E. \& Goebel, B. M. (1994). Taxonomic note: a place for DNA-DNA reassociation and $16 \mathrm{~S}$ rRNA sequence analysis in the present species definition in bacteriology. Int J Syst Bacteriol 44, 846-849.
Summanen, P. H., Durmaz, B., Väisänen, M. L., Liu, C., Molitoris, D., Eerola, E., Helander, I. M. \& Finegold, S. M. (2005). Porphyromonas somerae sp. nov., a pathogen isolated from humans and distinct from Porphyromonas levii. J Clin Microbiol 43, 4455-4459.

Thompson, J. D., Higgins, D. G. \& Gibson, T. J. (1994). CLUSTAL W: improving the sensitivity of progressive multiple sequence alignment through sequence weighting, position-specific gap penalties and weight matrix choice. Nucleic Acids Res 22, 4673-4680.

Willems, A. \& Collins, M. D. (1995). Reclassification of Oribaculum catoniae (Moore and Moore 1994) as Porphyromonas catoniae comb. nov., and emendation of the genus Porphyromonas. Int J Syst Bacteriol $45,578-581$. 